\title{
A Histopathological Scoring and Grading System for Renal Non-aa Amyloidosis: Prediction of Clinical Manifestations and Outcomes
}

Aygul Celtik ( $\square$ aygul.celtik@ege.edu.tr)

Ege University

\section{Sait Sen}

Ege University

Fatma Keklik Karadag

Ege University

Guray Saydam

Ege University

Gulay Asci

Ege University

\section{Banu Sarsik}

Ege University

Mehmet Ozkahya

Ege University

Huseyin Toz

Ege University

\section{Research Article}

Keywords: AL amyloidosis, grading, kidney biopsy, prognosis, unclassified amyloidosis

Posted Date: February 15th, 2021

DOl: https://doi.org/10.21203/rs.3.rs-194799/v1

License: (9) (1) This work is licensed under a Creative Commons Attribution 4.0 International License. Read Full License 


\section{Abstract}

Background: Kidney involvement is a common manifestation that contributes to morbidity and mortality in systemic amyloidosis. Amyloid load can be predicted by histopathological grading of amyloid deposits in kidney biopsy specimens. Our aim was to determine the relationship of renal amyloid deposition grade with clinical manifestations and outcomes in patients with biopsy proven renal non-AA amyloidosis.

Methods: This retrospective cohort study included 74 subjects with renal non-AA amyloidosis (52 AL amyloidosis and 22 unclassified amyloidosis). Baseline characteristics and follow-up data were recorded. Pattern and quantity of amyloid deposition in glomeruli, interstitium, vessels and tubulointerstitial changes were scored. Renal Amyloid Prognostic Score (RAPS) was obtained by addition of all scores and divided into three grades (RAPS grade I, II, III).

Results: In AL amyloidosis group, median follow-up was 11 (4-45 months). Among 52 patients, 17 received autologous stem cell transplantation and 29 received bortezomib based chemotherapy. Baseline eGFR was significantly lower among patients with RAPS grade III. Renal survival was significantly lower among patients with RAPS grade III. Patient survival was not significantly different according to RAPS grade. Type of amyloid could not be determined in 22 patients. In this group, baseline eGFR was significantly lower in patients with RAPS grade III.

Conclusions: In patients with AL amyloidosis, baseline kidney functions are associated with RAPS grade. Renal survival is significantly lower in patients with highest RAPS grade. However, patient survival is not significantly different according to RAPS grade.

\section{Introduction}

Amyloidosis is characterized by deposition of insoluble amyloid fibrils in various organs (1). The most common types are immunoglobulin related amyloidosis and AA amyloidosis. Immunoglobulin related amyloidosis is caused by malignant plasma cell clone producing amyloidogenic light chains (AL amyloidosis) and rarely heavy chain (AH amyloidosis) or both (AHL) (2). Classification of renal amyloidosis is based on these precursor proteins. The most definitive methods for detection of amyloid type are immunofluorescence or immunohistochemistry. However, accurate identification of amyloid types could not be done in $3-16 \%$ of cases using these traditional methods $(3,4)$. For these cases, laser microdissection/mass spectrometry is used in some tertiary centers. These cases were mainly diagnosed AL, AA and ALECT (leukocyte chemotactic factor 2) after evaluation with mass spectrometry (3). In our country, AA amyloidosis is relatively more common among patients with systemic amyloidosis when compared to AL amyloidosis. Underlying reason may be higher incidence of rheumatologic diseases (particularly FMF) and chronic infections, as well as ethnic differences $(5,6)$.

Amyloidogenic precursor proteins aggregate extracellularly, disturb structure of tissues and subsequently functions of affected organs. Type of affected organ and degree of amyloid accumulation may determine clinical manifestations and outcomes (7). Kidney involvement is common and presents with 
proteinuria and renal failure. It is a major determinant of morbidity and mortality (8). Relationship between amount and localization of amyloid deposits and clinical findings, morbidity and mortality were evaluated previously. Results were conflicting due to a lack of standardized methods for assessment of renal involvement (9-12).

In order to standardize histopathological evaluation and grading of renal amyloidosis, a scoring and grading system was defined to evaluate pattern and quantity of amyloid deposition in each compartment of kidney together with tubulointerstitial changes (6). In this study, our aim was to investigate the association of baseline clinical manifestations and outcomes with the renal amyloid prognostic score and grade in patients with renal non-AA amyloidosis.

\section{Material And Methods}

Our study is a retrospective cohort study which included subjects with biopsy-proven renal non-AA amyloidosis. All native kidney biopsies obtained between January 2005 and December 2019 were retrospectively reviewed. We identified 92 subjects with renal non-AA amyloidosis. Of 92 subjects, 18 patients were excluded: 14 were referred from other hospitals and 4 had inadequate data. Demographic and laboratory findings at the time of renal biopsy, treatment regimens and outcome data were collected from electronic medical reports.

All kidney biopsy specimens were obtained by ultrasound guided needle-biopsy, embedded in paraffin and cut into 4-6 $\mu \mathrm{m}$ sections. The sections were stained with hematoxylin and eosin, periodic acidSchiff, Masson's trichrome, Jones methamine silver and Congo red and evaluated by light microscopy (LM). For immunohistochemical (IHC) evaluation, paraffin-embedded tissue sections were stained with antibodies to amyloid A, fibrinogen alpha, transthyretin, kappa, lambda and lysosome by a fully automated device (Benchmark XT, Ventana Medical Systems, Tucson, USA). Cryosections were stained with polyclonal FITC-conjugated antibodies to kappa, lambda, IgG, $\lg A, \lg \mathrm{M}, \mathrm{C} 1 \mathrm{q}, \mathrm{C} 3 \mathrm{c}$ and fibrinogen (1/20 dilution, DAKO, Glostrup, Denmark) for immunoflourescence microscopy (IF).

Congo red stain was performed for detection of amyloid deposits and assessed by LM. Amyloid was identified as eosinophilic deposits which demonstrated apple-green birefringence under polarized light. AL amyloidosis was determined by IF, IHC and laboratory findings. Unclassified (UC) amyloidosis was defined when IHC and IF revealed equivocal or inconclusive results together with laboratory finding (Figure). All biopsies were evaluated according to the renal amyloidosis scoring and grading system previously described by Sen and Sarsik (6). Pattern and quantity of amyloid deposition in glomeruli, interstitium, vessels and tubulointerstitial changes were scored (Supp Data). By addition of all scores of glomerular, vascular and tubulointerstitial involvement, a renal amyloid prognostic score (RAPS) was obtained.

All patients with AL amyloidosis underwent bone marrow aspiration and biopsy with Jamshidi needles from iliac crest. After fixation with Hollant, bone marrow biopsy specimen were stained with haematoxylin and eosin, congo red and periodic acid-Schiff reagent. Immunohistochemical analysis 
done with Ventana automatic machine for kappa, lambda and immunoglobulins. Plasma cell monoclonality was defined as a $\mathrm{k} / \lambda$ ratio of more than 3 ( $\mathrm{k}$ clone) or less than 1 ( $\lambda$ clone). Plasma cell percentages were assessed on immunoperoxidase-stained sections for $\mathrm{k}$ and $\lambda$ immunoglobulins and Wright-Giemsa-stained aspirate smears. Amyloid deposits were stained Congo red and detected by apple-green birefringence under polarized light. Multiple myeloma was diagnosed according to International Myeloma Working Group criteria (13).

Estimated glomerular filtration rate (eGFR) was calculated by using CKD-EPI Eq. (14). End stage kidney disease (ESKD) was defined as requirement of renal replacement therapy (RRT).

Primary outcomes were kidney and patient survival. Follow-up period was defined as duration from kidney biopsy to last visit or death. Renal survival was calculated from date of biopsy to the initiation of RRT. Patients who died without requiring RRT were censored for analysis of renal survival (deathcensored renal survival).

Categorical variables were presented as percentage and compared with the chi-square test. Normality of distribution were assessed using the Kolmogorov-Smirnov test. Continuous variables were presented as mean \pm standard deviation or median and interquartile ranges. Continuous variables were compared with one-way ANOVA or Mann-Whitney $U$ test according to distribution of normality. Ordinary regression analysis was performed for estimating of relationship between age, gender, baseline eGFR, proteinuria and RAPS grade. Kaplan-Meier analysis was used for evaluation of death-censored renal survival and patient survival. A p value $<0.05$ was statistically significant. All statistics were performed with Statistical Package of Social Science (SPSS) software version 14.0 for Windows.

\section{Results}

Among 74 patients, 52 had AL amyloidosis. In 22 patients, amyloid deposition was unclassified (UC amyloidosis) Baseline characteristics and clinical findings are presented on Table 1.

\section{AL amyloidosis}

\section{Baseline clinical findings}

Mean age was $60 \pm 10$ years. Twenty-five patients were male. Median eGFR and proteinuria was 66 (2499) $\mathrm{ml} / \mathrm{min} / 1.73 \mathrm{~m}^{2}$ and 6.0 (3.7-9.8) g/day, respectively (Table 2).

All patients had a bone marrow biopsy. All except 2 patients were diagnosed AL amyloidosis by a kidney biopsy initially and bone marrow evaluation was done subsequently. Thirteen patients diagnosed multiple myeloma. Amyloid deposits were also detected in bone marrow biopsies of 34 patients.

Patients were divided into 3 groups according to RAPS grade. At the time of diagnosis, baseline eGFR were significantly lower among patients with RAPS grade III when compared to patients with RAPS grade I ( $p=0.002)$. Serum albumin and proteinuria were not significantly different according to RAPS grades 
$(p=0.913$ and $p=0.098$, respectively). In ordinary regression analysis adjusted by age, gender and proteinuria; eGFR was significantly associated with RAPS grade III (HR=0.97, 95\% Cl 0.95-0.99, $p=0.009)$.

\section{Follow-up data}

Seventeen patients had autologous stem cell transplantation (ASCT) with melphalan based conditioning regimen (melphalan $200 \mathrm{mg} / \mathrm{m}^{2}$ or $140 \mathrm{mg} / \mathrm{m}^{2}$ day -1) and 29 patients were treated with bortezomib based regimens. Six patients could not receive chemotherapy due to comorbidities and poor performance status.

Median follow-up was 11 (4-45) months. Twelve patients developed ESKD at a median of 7 (2-28) months and all underwent hemodialysis. Median baseline eGFR was $35(10-83) \mathrm{ml} / \mathrm{min} / 1.73 \mathrm{~m}^{2}$ for patients who developed ESKD and 68 (36-103) $\mathrm{ml} / \mathrm{min} / 1.73 \mathrm{~m}^{2}$ for patients did not develop ESKD $(p=0.041)$. RAPS grade was III in $13 \%$ of patients who did not develop ESKD and in $50 \%$ of patients who progressed to ESKD.

Death censored renal survival rates were $64 \%, 77 \%$ and $45 \%$ for RAPS grade I, II and III, respectively (Figure 2). Renal survival rate was significantly lower among patients with RAPS grade III when compared to those with RAPS grade I and II $(p=0.002)$. Patients who underwent ASCT had better renal survival when compared to those who received CT $(p=0.049)$.

Patient survival rates were $37 \%, 25 \%$ and $24 \%$ for RAPS grade I, II and III during follow-up, respectively $(p=0.575)$. Survival of patients who underwent ASCT was better than those who received bortezomib based regimens $(p=0.013)$.

\section{$\underline{\text { UC amyloidosis }}$}

In 22 patients, amyloid deposits were not classified. Mean age was $60 \pm 14$ years. Ten patients were male. At the time of biopsy, median serum creatinine and proteinuria were $3.66(1.52-5.84) \mathrm{mg} / \mathrm{dL}$ and 6.0 (4.2-9.9) g/d, respectively (Table 3). Baseline eGFR was significantly lower among patients with RAPS grade III $(p=0.012)$. Baseline proteinuria levels were not significantly different according to RAPS grade $(p=0.227)$.

Median follow-up was 23 (7-53) months. Two patients required RRT among 7 patients with RAPS grade II. Out of 15 patients with RAPS grade III, 14 underwent RRT. Fourteen patients started RRT within two months and one patient 25 months after diagnosis. Patient survival was $59.2 \%$ at $1^{\text {st }}$ year and $24.8 \%$ at $5^{\text {th }}$ year.

\section{Discussion}

We retrospectively evaluated clinical findings and outcomes of patients with biopsy proven renal non-AA amyloidosis (52 AL and 22 unclassified amyloidosis). Relationship between these parameters and a 
histopathological scoring and grading system was assessed. We demonstrated that increased RAPS grade was correlated with lower baseline eGFR but not with baseline proteinuria in patients with AL amyloidosis. Renal survival was associated with severity of renal amyloidosis, whereas patient survival was not, in AL amyloidosis group.

In amyloidosis, amyloidogenic precursors undergo extracellular misfolding and aggregation into amyloid fibrils in affected organs. Rate of aggregation depends on plasma concentration of precursor proteins which is determined by secretion and turnover. Amyloid deposits lead to malfunctioning of organs primarily by disturbing the structure of tissues $(7,15)$. Therefore, amyloid load which can be defined as degree, pattern and localization of amyloid deposition in kidney biopsy specimen may correlate with the severity of clinical manifestations. In our study, we evaluated renal amyloid load according to a histopathological scoring and grading system. The grading system was associated with renal function and outcome in patients with AA amyloidosis (16). Baseline eGFR was lower in patients with RAPS grade III, however proteinuria was not different according to RAPS grade in AL amyloidosis group. In some previous studies, amyloid load was associated with the degree of renal failure and proteinuria at the time of diagnosis. Amyloid-positive area in renal tissue was associated with renal function in patients with AL amyloidosis (11). Yao et al found that the amount of glomerular AL amyloid was positively associated with proteinuria and kidney failure (12).

In retrospective studies, baseline eGFR level and proteinuria was associated with outcomes in AL amyloidosis. Castano et al showed that severe glomerular amyloid deposition is indicative of poor patient survival among 35 patients with AA and AL amyloidosis (9). The risk for overall death increased as the renal amyloid load increased (12). An overall amyloid score was strongly correlated with development of ESKD among 39 patients with renal AL amyloidosis (17). Recently, two clinical renal staging systems based on eGFR and proteinuria were validated for AL amyloidosis. Both demonstrated that decreased eGFR and increased proteinuria were predictors of poor renal outcome. However, renal staging was not significantly associated with patients' survival (18-20). It is reasonable to assume that renal amyloid load may be correlated with severity of renal staging and be a predictor of renal and patient survival. Histopathological grading of amyloid load can be interpreted easily by evaluation of kidney biopsies. However, histopathological evaluation of kidney biopsies has not been incorporated into previous clinical staging systems for AL amyloidosis $(18,19)$. In our study, RAPS grade includes overall amyloid load and tubulointerstitial changes. Patients with highest RAPS grade had worst renal survival. At the time of diagnosis, renal histopathological amyloid grading may be useful for predicting kidney outcomes along with clinical and laboratory findings. In contrast, we did not demonstrate a relationship with patient survival, indicating that degree of amyloid burden in kidney does not have a major prognostic impact in survival of patients with AL amyloidosis. In AL amyloidosis, patient outcome is mainly influenced by many factors such as age, co-morbidities, extrarenal organ involvement like heart and treatment modalities.

Classification of systemic amyloidosis is based on the precursor proteins and essential for treatment and prognosis. The most definitive methods for determination of amyloid type are IHC and IF but distinction 
between amyloid types may still be difficult in some cases. IF staining can be negative for immunoglobulins with deleted or modified epitopes (21). Additionally, amyloid deposits occasionally exhibit nonspecific immunostaining due to contamination with serum proteins, interaction of reagent and amyloid proteins or humoral reaction against amyloid fibrils $(22,23)$. In previous studies, amyloid type could not be determined in $3-16 \%$ of cases $(3,4)$. In our cohort, IF, IHC, laboratory findings and story of patients were inadequate to determine amyloidosis type in 22 patients. In a previous study, $15.6 \%$ of cases were evaluated with mass spectrometry and $45 \%$ of them had AL, $17 \%$ ALECT2 and $12 \%$ AA amyloidosis (3). Immunoglobulin related or AA amyloidosis are likely to account for most cases in the UC group. RAPS grade was III in nearly $70 \%$ of patients with UC amyloidosis, suggesting that they were diagnosed at an advanced stage. Therefore, 14 of them required RRT within two months after diagnosis.

This study has several limitations. Small sample size and retrospective design are main limitations. We could not classify amyloidosis in 22 patients because we did not have mass spectrometry which is gold standard for typing of amyloidosis. We were unable to diagnose ALECT2 amyloidosis in our study.

Patients with advanced glomerular amyloid deposits might have AFib amyloidosis but fibrinogen staining was negative. In addition, interstitial dominant amyloid deposits compatible with AApo amyloidosis was not detected in our cohort.

In conclusion, degree of amyloid load in kidney is associated with baseline eGFR in patients with AL amyloidosis. RAPS grade is significantly associated with progression to ESKD but not associated with patients' survival in AL amyloidosis. Histopathological findings in kidney biopsy may be considered for prediction of renal survival in AL amyloidosis patients with kidney involvement.

\section{Declarations}

\section{Ethics approval and consent to participated}

We preformed all the biopsies and blood samples included in this study according to our clinical followup and biopsy protocol. No additional blood samples or biopsy were taken for the purpose of this retrospective study. Therefore, informed consent was not obtained from any individuals included in the study and approval of the ethics committee was not required.

\section{Consent for publication}

Consent for publication was not obtained from any individuals included in the study due to the retrospective design.

\section{Availability of data and materials}

The datasets generated and analysed during the current study are not publicly available for the privacy of partcipants but are available from the corresponding author (A.C.) on reasonable request.

\section{Competing interests}


The authors declare no competing financial interests. The authors hereby confirm that neither the manuscript nor any part of it has been published or is being considered for publication elsewhere, except in abstract form.

\section{Funding}

The study was not supported by any funding.

\section{Authors' contributions}

The authors acknowledge that they participated sufficiently in the work to take public responsibility for its content. All authors gave final approval of the version to be published.

AC: She designed the study, collected data, performed the analysis, wrote the article.

SS: He performed pathological analysis of the renal biopsies and wrote the article.

FKK: She collected data, evaluated patients and performed the analysis.

GS: He evaluated patients, performed the analysis and wrote the article.

GA: She collected data, evaluated patients, performed the analysis and wrote the article.

BS: She performed pathological analysis of renal biopsies and participated data collection.

MO: He participated in study design and writing of the article.

HT: He designed the study, performed the analysis and wrote the article.

\section{Acknowledgements}

None

\section{References}

1. Merlini G, Bellotti V. Molecular mechanisms of amyloidosis. N Engl J Med. 2003;349(6):583-96.

2. Khalighi MA, Dean Wallace W, Palma-Diaz MF. Amyloid nephropathy. Clin Kidney J. 2014;7(2):97106.

3. Said SM, Sethi S, Valeri AM, Leung N, Cornell LD, Fidler ME, et al. Renal amyloidosis: origin and clinicopathologic correlations of 474 recent cases. Clinical journal of the American Society of Nephrology : CJASN. 2013;8(9):1515-23.

4. von Hutten $\mathrm{H}$, Mihatsch M, Lobeck $H$, Rudolph B, Eriksson M, Rocken C. Prevalence and origin of amyloid in kidney biopsies. Am J Surg Pathol. 2009;33(8):1198-205.

5. Tuglular S, Yalcinkaya F, Paydas S, Oner A, Utas C, Bozfakioglu S, et al. A retrospective analysis for aetiology and clinical findings of 287 secondary amyloidosis cases in Turkey. Nephrol Dial 
Transplant. 2002;17(11):2003-5.

6. Sen S, Sarsik B. A proposed histopathologic classification, scoring, and grading system for renal amyloidosis: standardization of renal amyloid biopsy report. Arch Pathol Lab Med. 2010;134(4):53244.

7. Dember LM. Amyloidosis-associated kidney disease. J Am Soc Nephrol. 2006;17(12):3458-71.

8. Gertz MA, Leung N, Lacy MQ, Dispenzieri A, Zeldenrust SR, Hayman SR, et al. Clinical outcome of immunoglobulin light chain amyloidosis affecting the kidney. Nephrol Dial Transplant. 2009;24(10):3132-7.

9. Castano E, Palmer MB, Vigneault C, Luciano R, Wong S, Moeckel G. Comparison of amyloid deposition in human kidney biopsies as predictor of poor patient outcome. BMC Nephrol. 2015;16:64.

10. Gillmore JD, Lovat LB, Persey MR, Pepys MB, Hawkins PN. Amyloid load and clinical outcome in AA amyloidosis in relation to circulating concentration of serum amyloid A protein. Lancet. 2001;358(9275):24-9.

11. Kuroda T, Tanabe N, Kobayashi D, Wada Y, Murakami S, Nakano M, et al. Significant association between renal function and amyloid-positive area in renal biopsy specimens in $\mathrm{AL}$ amyloidosis. BMC Nephrol.13:118.

12. Yao Y, Wang SX, Zhang YK, Qu Z, Liu G, Zou WZ. A clinicopathological analysis in a large cohort of Chinese patients with renal amyloid light-chain amyloidosis. Nephrol Dial Transplant. 2013;28(3):689-97.

13. Rajkumar SV, Dimopoulos MA, Palumbo A, Blade J, Merlini G, Mateos MV, et al. International Myeloma Working Group updated criteria for the diagnosis of multiple myeloma. The Lancet Oncology. 2014;15(12):e538-48.

14. Levey AS, Stevens LA, Schmid CH, Zhang YL, Castro AF, 3rd, Feldman HI, et al. A new equation to estimate glomerular filtration rate. Ann Intern Med. 2009;150(9):604-12.

15. Buxbaum JN, Linke RP. A molecular history of the amyloidoses. J Mol Biol. 2012;421(2-3):142-59.

16. Celtik A, Sen S, Keklik F, Saydam G, Asci G, Sarsik B, et al. A histopathological scoring and grading system to predict outcome for patients with AA amyloidosis. Int Urol Nephrol. 2020;52(7):1297-304.

17. Rubinstein S, Cornell RF, Du L, Concepcion B, Goodman S, Harrell S, et al. Novel pathologic scoring tools predict end-stage kidney disease in light chain (AL) amyloidosis. Amyloid. 2017;24(3):205-11.

18. Kastritis E, Gavriatopoulou M, Roussou M, Migkou M, Fotiou D, Ziogas DC, et al. Renal outcomes in patients with AL amyloidosis: Prognostic factors, renal response and the impact of therapy. American journal of hematology. 2017;92(7):632-9.

19. Palladini G, Hegenbart U, Milani P, Kimmich C, Foli A, Ho AD, et al. A staging system for renal outcome and early markers of renal response to chemotherapy in AL amyloidosis. Blood. 2014;124(15):2325-32. 
20. Zhu Z, Yue C, Sun Y, Li X, Li M. Light-chain amyloidosis with renal involvement: renal outcomes and validation of two renal staging systems in the Chinese population. Amyloid. 2019;26(4):186-91.

21. Novak L, Cook WJ, Herrera GA, Sanders PW. AL-amyloidosis is underdiagnosed in renal biopsies. Nephrol Dial Transplant. 2004;19(12):3050-3.

22. Verine J, Mourad N, Desseaux K, Vanhille P, Noel LH, Beaufils H, et al. Clinical and histological characteristics of renal AA amyloidosis: a retrospective study of 68 cases with a special interest to amyloid-associated inflammatory response. Hum Pathol. 2007;38(12):1798-809.

23. Kebbel A, Rocken C. Immunohistochemical classification of amyloid in surgical pathology revisited. Am J Surg Pathol. 2006;30(6):673-83.

\section{Tables}

Table 1. Baseline clinical findings and follow-up data of all patients

$$
\mathrm{AL} \text { amyloidosis }(\mathrm{n}=52) \quad \text { UC amyloidosis }(\mathrm{n}=22)
$$

Baseline characteristics

\begin{tabular}{lll} 
Age (years) & $60 \pm 10$ & $60 \pm 14$ \\
\hline Gender $($ male) $(\mathrm{n}, \%)$ & $25(48 \%)$ & $10(45.4 \%)$ \\
\hline Serum creatinine $(\mathrm{mg} / \mathrm{dL})$ & $1.02(0.74-2.42)$ & $3.66(1.52-5.84)$ \\
\hline eGFR $\left(\mathrm{ml} / \mathrm{min} / 1.73 \mathrm{~m}^{2}\right)$ & $66(24-99)$ & $14(10-37)$ \\
\hline Serum albumin $(\mathrm{mg} / \mathrm{dL})$ & $2.5(2.3-3.1)$ & $2.5(2.1-3.1)$ \\
\hline Proteinuria $(\mathrm{g} /$ day $)$ & $6.0(3.7-9.8)$ & $6.0(4.2-9.9)$ \\
\hline Outcome & & $23(7-53)$ \\
Follow-up (months) & $11(4-45)$ & $68.1 \%$ \\
\hline Requirement of RRT & $23.1 \%$ & $24.8 \%$ \\
\hline Patient survival at $5^{\text {th }}$ year & $30.9 \%$ &
\end{tabular}

Data are expressed as mean \pm standard deviation, median (interquartile range), $n(\%)$. AL amyloidosis, Light-chain amyloidosis. UC amyloidosis, unclassified amyloidosis. 
Table 2. Baseline demographical features and clinical findings of patients with AL amyloidosis

\begin{tabular}{|c|c|c|c|c|}
\hline & $\begin{array}{l}\text { RAPS grade I } \\
(n=15)\end{array}$ & $\begin{array}{l}\text { RAPS grade II } \\
(n=26)\end{array}$ & $\begin{array}{l}\text { RAPS grade III } \\
(n=11)\end{array}$ & $\mathrm{p}$ \\
\hline Age (years) & $58 \pm 12$ & $60 \pm 10$ & $63 \pm 9$ & 0.479 \\
\hline Gender (male) (n, \%) & $8(53.3 \%)$ & $10(38.4 \%)$ & $7(63.6 \%)$ & 0.334 \\
\hline $\begin{array}{l}\text { Serum creatinine } \\
(\mathrm{mg} / \mathrm{dL})\end{array}$ & $0.89(0.70-1.18)$ & $0.94(0.70-2.93)$ & $2.28(1.26-3.95)$ & $0.024^{*}$ \\
\hline $\begin{array}{l}\text { eGFR }(\mathrm{ml} / \mathrm{min} / 1.73 \\
\left.\mathrm{m}^{2}\right)\end{array}$ & $91(49-106)$ & $67(22-101)$ & $27(11-66)$ & $0.029 \S$ \\
\hline $\begin{array}{l}\text { Serum albumin } \\
(\mathrm{mg} / \mathrm{dL})\end{array}$ & $2.5(2.3-3.2)$ & $2.6(2.2-3.1)$ & $2.5(1.8-3.1)$ & 0.913 \\
\hline Proteinuria (g/day) & $5.9(4.4-11.4)$ & $5.2(1.5-8.3)$ & $8.3(5.0-13.6)$ & 0.098 \\
\hline \multicolumn{5}{|c|}{${ }^{*} p=0.002$ for grade I vs III, $\S_{p}=0.005$ for grade I vs III } \\
\hline \multicolumn{5}{|c|}{$\begin{array}{l}\text { Data are expressed as mean } \pm \text { standard deviation, median (interquartile range), } n(\%) \text {. AL amyloidosis, } \\
\text { Light-chain amyloidosis. }\end{array}$} \\
\hline
\end{tabular}

Table 3. Baseline demographical features and clinical findings of patients with UC amyloidosis

\begin{tabular}{|c|c|c|c|}
\hline & RAPS grade II $(n=7)$ & RAPS grade III $(n=15)$ & $\mathrm{p}$ \\
\hline Age (years) & $58 \pm 13$ & $60 \pm 15$ & 0.763 \\
\hline Gender (male) (n, \%) & $5(71.4 \%)$ & $5(33.3 \%)$ & 0.095 \\
\hline Serum creatinine $(\mathrm{mg} / \mathrm{dL})$ & $1.59(0.90-4.16)$ & $3.89(2.92-6.18)$ & 0.032 \\
\hline eGFR $\left(\mathrm{ml} / \mathrm{min} / 1.73 \mathrm{~m}^{2}\right)$ & $35(16-90)$ & $14(9-21)$ & 0.012 \\
\hline Serum albumin (mg/dL) & $2.2(1.9-2.4)$ & $2.8(2.1-3.2)$ & 0.083 \\
\hline Proteinuria (g/day) & $8.4(5.1-10.3)$ & $5.1(3.8-9.0)$ & 0.227 \\
\hline
\end{tabular}

\section{Figures}




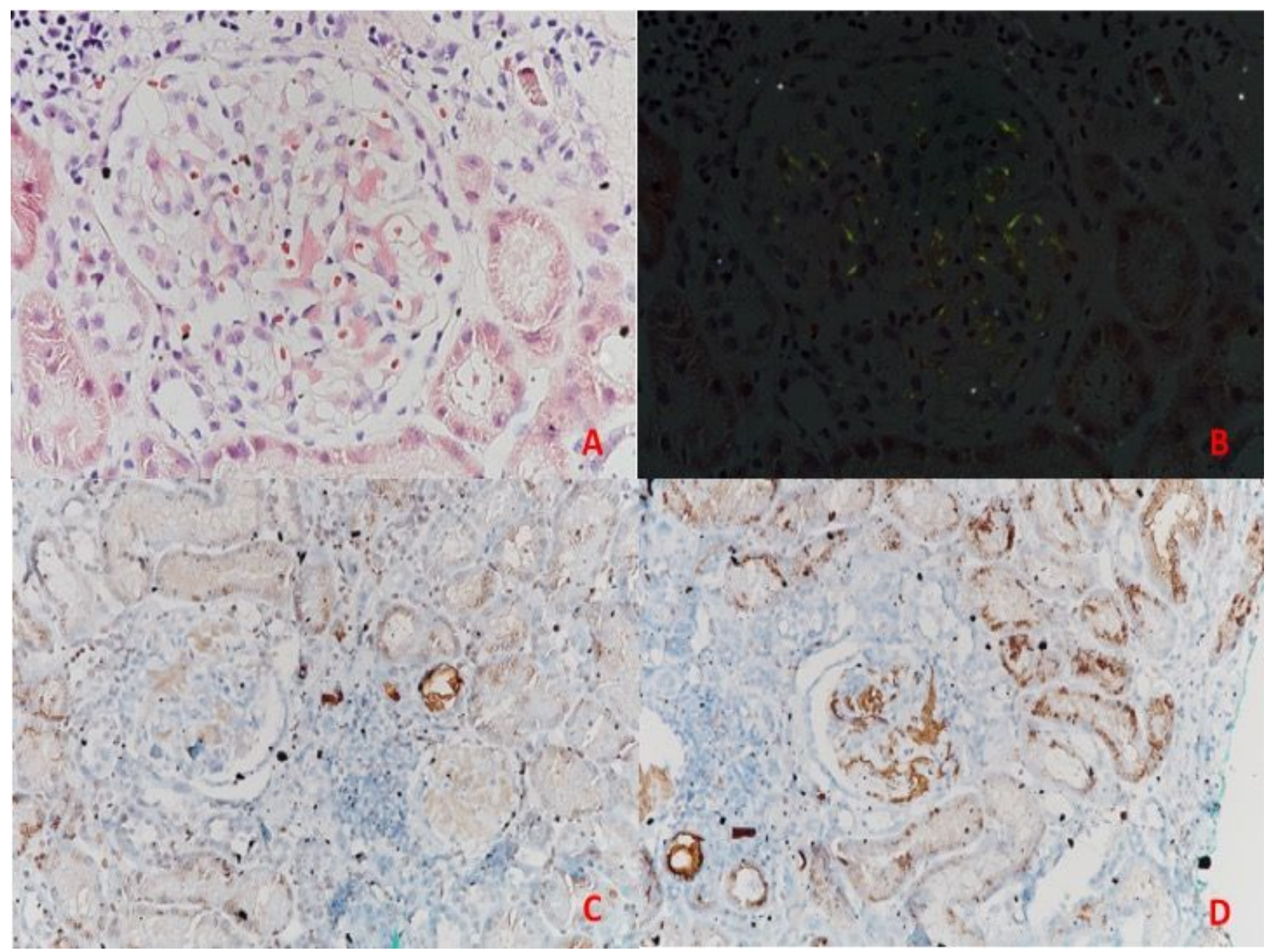

Figure 1

Glomerular amyloid deposits are stained with Congo red (A) and show apple-green birefringence under polarized light (B). Immunohistochemical stain for kappa light chain is negative (C) and positive with for lambda chain (D). All images were taken at 200x. 


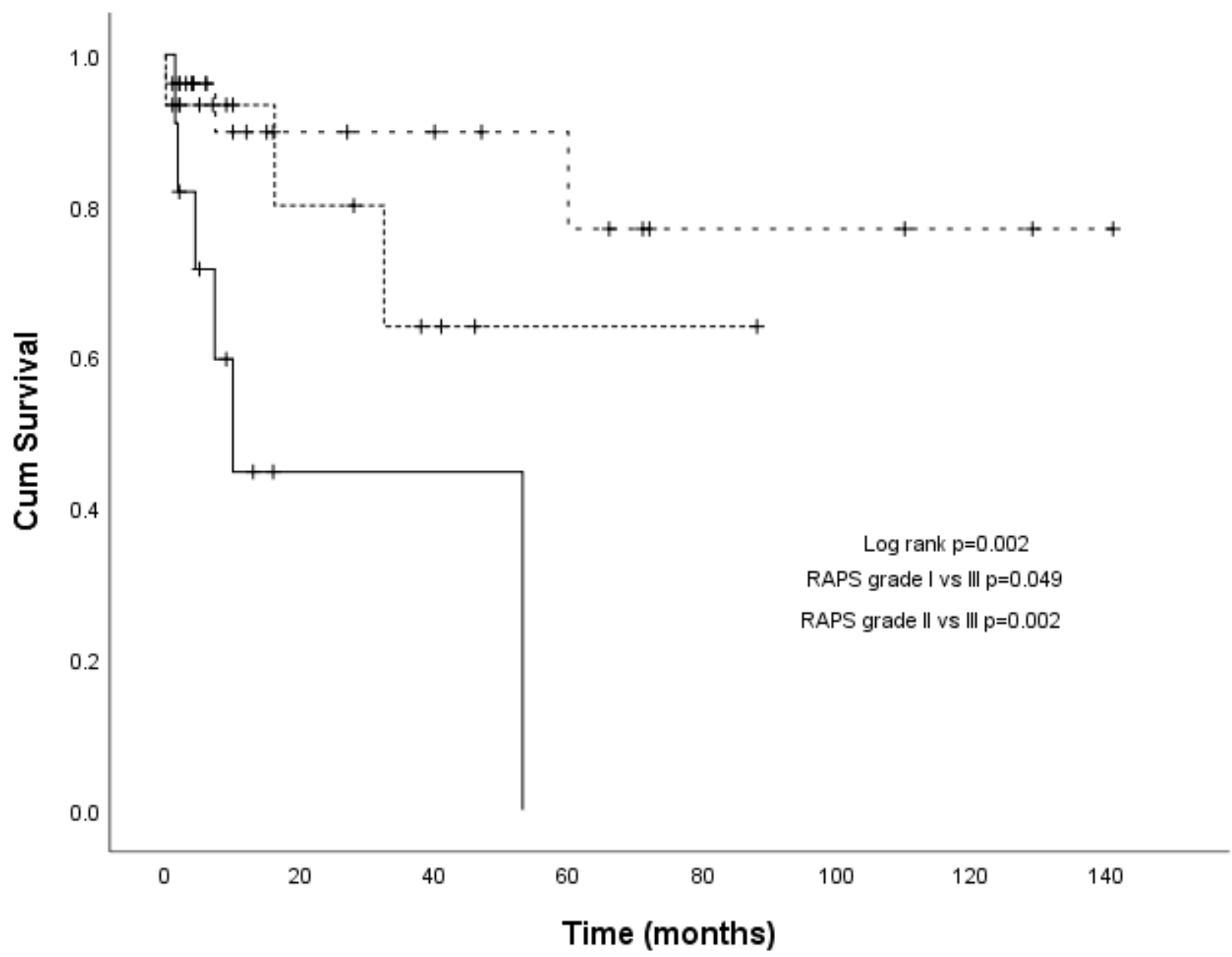

Figure 2

Death censored renal survival according to RAPS grades in AL amyloidosis group

\section{Supplementary Files}

This is a list of supplementary files associated with this preprint. Click to download.

- CeltiketalSuppdoc.docx 